\title{
Intracranial Metastasis as the Initial Presentation of a Young Woman with Luminal B Her-2 Positive Stage 4 Breast Cancer
}

\author{
Santi Christiani Gultom, Amaylia Oehadian \\ Division of Hematology and Medical Oncology, Department of Internal Medicine, Faculty of Medicine Universitas \\ Padjadjaran-Dr. Hasan Sadikin General Hospital Bandung, Indonesia
}

\begin{abstract}
Objective: Breast cancer is the second most common cause of brain metastasis (BM) among all of the solid cancers, with metastases occurring in $10 \%-16 \%$ of patients and in as many as $30 \%$ of autopsy studies. Breast cancer-related BM usually has a poor prognosis and survival rate in the absence of any treatment within 2 months. Survival after BM is related to the subtype of the primary tumor. Human epidermal growth factor-2 (HER-2)-positive patients have a significantly better prognosis compared with other subtypes. The prognosis for the majority of patients with $\mathrm{BM}$ remains poor, despite local and systemic therapies, with a median survival of around 10 months.
\end{abstract}

Methods: This case is interesting because our patient is very young, diagnosed with BM before breast cancer was identified, bit still surviving 12 months after her BM diagnosis. A 19-year old woman presented with seizures, vomit and headaches.

pISSN: 2302-1381;

eISSN: 2338-4506;

http://doi.org/10.15850/

ijihs.v8n1.1911

IJIHS. 2020;8(1):43-6

Received:

December 16, 2019

Accepted:

March 9, 2020
Results: A cranial CT-scan showed an intracranial mass. The intracranial tumor was removed, and yielded a histopathological result of metastatic adenocarcinoma. Further examination found a lump in her right breast. She was diagnosed with intracranial metastatic stage 4 luminal B Her 2(+) breast cancer. She was referred for WBRT, a mastectomy, chemotherapy with docetaxel cyclophosphamide 4 cycles, followed by 12 cycles of trastuzumab, and continued treatment with tamoxifen and goserelin. The last PET-Scan showed no residual disease.

Conclusion: Breast cancer as the primary tumor should be considered in women with a metastatic brain tumor. With appropriate treatment, even stage IV luminal B breast cancer with BM can still have a long life with good quality.

Keywords: Breast cancer, brain metastasis, central nervous system (CNS)

\section{Introduction}

Advanced Breast Cancer (ABC) comprises both locally advanced breast cancer (LABC) and metastatic breast cancer (MBC). Although treatable, MBC remains an incurable disease with a median overall survival (OS) of three years and a 5-years survival of only $25 \%$. The

Correspondence:

Santi Christiani Gultom,

Division of Hematology and Medical Oncology,

Department of Internal Medicine, Faculty of Medicine

Universitas Padjadjaran-Dr. Hasan Sadikin General

Hospital Bandung, Indonesia

e-mail: santich.gultom@gmail.com development of brain metastases (BM) is one of the major challenges for patients with stage III and IV breast cancer. In a recent study, brain metastases-free survival differed significantly between breast cancer subtypes and was shortest in patients with triple receptornegative cancer. Central nervous system (CNS) metastases account for the majority of malignant brain tumors, and may appear either within the brain parenchyma or along the leptomeninges. Breast cancer is the second most common cause of CNS metastases, and is the solid tumor most commonly associated with leptomeningeal metastases. ${ }^{1,2}$

On the basis of case series from the 1960s 
and 1970s, the incidence of clinically evident brain metastases among women with stage IV breast cancer is estimated to be $10 \%$ to $16 \%$. These figures underestimate the true incidence, given that brain metastases are found in $30 \%$ of patients at autopsy. Parenchymal brain metastases typically follow a vascular distribution, consistent with a mechanism of hematogenous spread. Supratentorial lesions are more common than infratentorial lesions, and there is a predilection for vascular border zones and the gray and white matter junction. In contrast, leptomeningeal metastases arise via multiple pathways, including hematogenous spread, direct extension, and infiltration from vertebral metastases via Batson's plexus. Involvement of the leptomeninges is less common clinically than parenchymal metastases, and is found in $5 \%$ to $16 \%$ of patients at autopsy. ${ }^{3,4}$

Young age appears to be a risk factor for the development of CNS metastases. For example, in an autopsy review of 1,044 patients with breast cancer, the median age of patients with CNS metastases was about 5 years younger than that of patients without CNS metastases. Several groups have also found an association between the hormone receptor status and the incidence of CNS relapse, although these studies generally have been small in scale. In one study of 217 women with primary breast cancer, estrogen receptor (ER)-negative breast cancers were more likely to metastasize to the brain ( $10 \%$ vs $4 \%$ clinical incidence). In an autopsy study of 25 patients, ER-negative tumors were also more likely to metastasize to the leptomeninges. Whether this observed association relates to the inherent biology of ER-negative tumors, the ability of hormonal therapy to penetrate the CNS (thereby providing a relative risk reduction for ER-positive tumors), and/or the inverse relationship between the hormone receptor and the HER2 status is unclear on the basis of the available evidence. The association of CNS metastases with the human epidermal growth factor receptor 2 (Her2) overexpression merits special mention. An amplification of HER2 is present in $25 \%$ to $30 \%$ of human breast cancers and correlates with diminished disease-free and overall survival. Among 319 women with primary breast cancer, HER2 overexpression was the strongest predictor of the site of the first relapse in a multivariate model, with a $4.3 \%$ vs $0.4 \%$ incidence of brain metastases. ${ }^{3,5}$

The most common presenting symptom of parenchymal brain metastases is a headache, occurring in $24 \%$ to $48 \%$ of patients. Other common manifestations include mental status changes and cognitive disturbances, which are present in $24 \%$ to $34 \%$ of patients. ${ }^{6}$

This case is interesting because our patient is very young, presenting with BM before her breast cancer diagnosis, but still surviving 21 months after her BM diagnosis.

\section{Case}

In April 2018, a 19-year-old woman came to our hospital with seizures, blurred eyes, headaches and vomiting. She consulted a neurosurgeon and underwent a brain CTscan on the $3^{\text {rd }}$ of April, 2018. The CT-scan revealed a multilobulated tumor mass with cystic components on the edges including right ventricular posterior pericocalus / right parietal lobe and the medial right occipital lobe (size $\pm 4.54 \times 3.88 \times 4.27 \mathrm{~cm}$ ) accompanied by extensive perifocal edema vitalizing the posterior right lateral and ventricular III ventricles and a mild midline shift to the left as much as $\pm 0.76 \mathrm{~cm}$ pushing the midbrain to anterior inferior, suggestive of a high-grade glioma. There were also multiple lacunae / ischemic degeneration lesions in the white frontal lobe white matter. There was no acute infarction, bleeding, vascular malformations or intracranial space occupying lesions. A craniotomy with a biopsy was performed. Histopathology showed metastatic of papillary adenocarcinoma with difficulty in ascertaining its origin. The patient was then treated with whole brain radiotherapy (WBRT). While undergoing WBRT, the patient was referred to medical oncology for further evaluation of the primary tumor. A palpable $4-\mathrm{cm}$ hard, painless mass was found in the lower right quadrant of her right breast. She had had a lump in her right breast since November 2017 but it had not been evaluated. She had had menarche at the age of 11 and her grandmother had suffered from breast cancer. A positron emission tomography (PET) scan was carried out and showed an irregular mass in the lower right quadrant of the medial right breast with central necrosis accompanied by metabolic activity at the periphery, highly suggestive of malignancy. A hypermetabolic lobulated mass in the right parietooccipital surrounded by the peripheral area of the edema and pressing the posterior to the right lateral ventricle is consistent for a metastasis. No hypermetabolic lesions were seen in the lymph nodes or other organs suggestive of malignancies / other metastases. A reevaluation of the intracranial 
biopsy tissue showed malignant tumor forming papillary structures section of the anaplastic cells with rounded and pleomorphic nuclei, hyperchromatic, a moderate amount of cytoplasm, the mitotic count was twenty two per ten high power field (22/10 hpf). The differential diagnosis were metastatic adenocarcinoma and anaplastic ependymoma.

She underwent an incisional biopsy on her breast lump. Breast histopathology showed a malignant breast tumor invading stroma and fat. The tumor was composed of anaplastic epithelial cells with marked pleiomorphic nuclei (score 3), coarse chromatin and prominent nuclei, the mitotic count was twenty five per ten hpf (score 3), arranged in solid clusters (tubular structures $<10 \%$, score $3)$, with necrosis in the central part of the tumor. There were ductal carcinoma insitu foci in peripheral parts of the tumor, mostly solid types with a few comedo types, high grade. Lymphovascular and perineural invasion were not found. Immunohistochemistry staining showed estrogen receptor (ER) positive of $80 \%$, weak to moderately stained, progesterone receptor (PR) positive of $5 \%$ weak stained, Her-2 +3 for the herceptest score and a Ki67 expression of 75\% (An invasive breast carcinoma of no special type pathological grade III luminal B, Her2 positive). The patient underwent a right mastectomy which showed metastasis in 1 of 5 axillary lymph nodes. She underwent 4 cycles of chemotherapy with docetaxel and cyclophosphamide and 12 cycles with trastuzumab. In September 2018, the patient continued therapy with tamoxifen. In October 2018, a PET-scan showed no residual disease. The patient continued treatment with tamoxifen and goserelin and has since remained in remission.

\section{Discussion}

Generally, patients having breast cancer brain metastases (BCBM) is associated with a poor prognosis. The outcome for these patients remains poor even after chemotherapy and radiotherapy. Most BCBM patients are characterized by rapid growing, hormone receptor negative and/or Her-2 positive tumors concurrent with visceral metastases. ${ }^{7}$ Breast cancer is divided into three distinct diseases with heterogeneous expression of estrogen receptor/progesteron receptor (ER/ PgR) and Her2 status. Luminal type cancers, which are ER/PgR positive without a Her2positive status, tend to be the most favorable for long-term survival. Whereas, the triple negative subtype (basal-like) and Her2positive tumors are the most sensitive to chemotherapy, but have the poorest prognosis. Of women diagnosed with advanced breast cancer, $10 \%$ to $16 \%$ develop central nervous system (CNS) metastases. Several risk factors have been associated with the development of CNS lesions in patients with $\mathrm{MBC}$, including being of a younger age ( $<50$ year), having more than two metastatic sites at MBC diagnosis, and being Her2-positive. Patients with Her2positive MBC tumors are 2 to 4 times more likely to develop CNS tumors than patients who are Her2-negative. The proportion of patients with brain metastases in triple-negative, Her2positive status and luminal subtypes was $28 \%$, $53 \%$ and $19 \%$, respectively. This indicates that triple-negative and Her2-positive breast cancer have a special predisposition to metastasize to the brain. ${ }^{8}$ In one study, Mauer C et al., found that the median OS for BC without BM was 46.7 months (IQR 23.0-145.6 months) and for BCBM patients 20.8 months (IQR 5.36not reached). Patients without CNS symptoms at the time of their BM diagnosis had a better OS than patients with clinical signs related to their BM (HR 0.49, 95\% CI 0.25 to 0.94). ${ }^{9}$

CNS involvement typically occurs late in the course of metastatic breast cancer. In most cases, the involvement of the lungs, liver, or bone precedes the diagnosis of CNS metastasis. ${ }^{10}$

In this case, CNS metastasis was the first discovery so the patient underwent WBRT. At present, there is no evidence that early detection of brain metastases alters the chances of survival or the quality of life of patients with breast cancer, although no prospective studies have specifically addressed this issue. Conversely, although corticosteroids, WBRT, surgery and stereotactic radiosurgery (SRS) can provide excellent palliation of some symptoms, motor loss and other focal deficits are frequently not fully reversible. Therefore, it is possible that early detection may improve the quality of life by identifying patients for treatment before irreversible neurologic damage has occured. For many years, chemotherapy and endocrine therapy have not been considered as playing a role in the treatment of patients with brain metastases because of the presence of the blood-brain barrier (BBB). Most chemotherapy agents used in the treatment of breast cancer either do not cross an intact BBB, or are pumped out of the CNS by phosphoglycoprotein in the endothelial cells comprising the BBB. They may not reach sufficient therapeutic levels to treat CNS 
metastases. The finding that chemotherapy and trastuzumab treatment are associated with improved survival even after adjusting for relevant variables in patients after the development of CNS metastases is interesting. The studies have proved the permeability of the blood-brain barrier in patients with brain metastases and in patients after WBRT. It has also been observed that systemic therapy carried out after WBRT prolongs survival due to the control of extracranial disease. Actually, after WBRT of brain metastases, the bloodbrain barrier is, in part, disrupted so that many chemotherapeutics and hormonal agents may reach a therapeutic level in tumors. ${ }^{11}$

Trastuzumab in combination with chemotherapy significantly improves OS and disease-free survival in Her2-positive BC.

\section{References}

1. Cardoso F, Costa A, Norton L. ESO-ESMO 2nd International Consensus Guidelines for advanced breast cancer (ABC2). Ann Oncol. 2014;25(10): 1871-88.

2. Cardoso F, Spence D, Mertz S, CorneliussenJames D, Sabelko K, Gralow J, et al. Global analysis of advanced/metastatic breast cancer: decade report (2005-2015). Breast. 2018;39:131-38.

3. Lin NU, Bellon JR, Winer EP. CNS metastases in breast cancer. 2004;22(17):3608-17.

4. Lee Y-T. Breast carcinoma: Pattern of metastasis at autopsy. J Surg Oncol. 1983;23:175-80.

5. Boogerd W, Vos VW, Hart AAM. Brain metastases in breast cancer; natural history, prognostic factors, and outcome. J Neuro Oncol. 1993;15:165-74.

6. Lassman AB, DeAngelis LM. Brain metastases. Neurol Clin. 2003;21(1):1-vii.

7. Cagney DN, Martin AM, Catalano PJ, Redig AJ, Lin NU, Lee EQ, et al. Incidence and prognosis of patients with brain metastases at diagnosis of systemic malignancy: a population-based study. Neuro Oncol. 2017;19(11):1511-21.

8. Weil RJ, Palmieri DC, Bronder JL, Stark AM, Steeg PS. Breast cancermetastasis to the central
The median overall survival (OS) for BCBM patients treated with whole brain radiotherapy (WBRT) alone is poor being of less than 6 months. More recent analyses suggest that the prognosis of BCBM patients, especially those with Her2-positive BCBM is improving which is probably a result of better systemic treatment options leading to better control of the disease outside the central nervous system (CNS). ${ }^{12,13}$

This case suggests that appropriate treatment with whole brain radiotherapy followed by trastuzumab combined with chemotherapy remains the best option for giving a long, good quality life to patients who present with brain metastasis in luminal B Her2-positive stage IV breast cancer.

nervous system. Am J Pathol. 2005;167:91320.

9. Maurer C, Tulpin L, Moreau M, Dumitrescu C, Azambuja Ed, Paesmans P, et al. Risk factors for the development of brain metastases in patients with HER2positive breast cancer. ESMO Open. 2018;3:e000440.

10. Engel J, Eckel R, Aydemir U, Aydemir S, Kerr J, Schlesinger-Raab A, et al. Determinants and prognoses of locoregional and distant progression in breast cancer. Int J Radiat Oncol Biol Phys. 2003;55(5):1186-195.

11. Lin NU, Bellon JR, Winer EP. CNS metastases in breast cancer. J Clin Oncol. 2004;22:3608-617.

12. Brufsky AM, Mayer M, Rugo HS, Tan CE, Tripathy $\mathrm{D}$, Tudor IC, et al. Central nervous system metastases in patients with HER2-positive metastatic breast cancer: incidence, treatment, and survival in patients from registHER. Clin Cancer Res. 2011;17(14):4834-43.

13. Dawood S, Broglio K, Esteva FJ, Buzdar AU, Hortobagyi GN, Giordano HG. Defining prognosis for women with breast cancer and CNS metastases by HER2 status. Ann Oncol. 2008;19(7):1242-48. 